\title{
An Electrochemical Investigation of Additive Effect in Trench-Filling of ULSI Interconnects by Electroless Copper Deposition
}

\author{
Madoka Hasegawa, ${ }^{a}$ Noriyuki Yamachika, ${ }^{\text {a }}$ Yutaka Okinaka, ${ }^{\mathrm{b}}$ Yosi Shacham-Diamand, \\ and Tetsuya OSAKA ${ }^{\mathrm{a}, \mathrm{b}}$
}

\begin{abstract}
a Department of Applied Chemistry, School of Science and Engineering, Waseda University (3-4-1 Okubo, Shinjukuku, Tokyo 169-8555, Japan)

b Advanced Research Institute for Science and Engineering, Waseda University (3-4-1 Okubo, Shinjuku-ku, Tokyo 169-8555, Japan)

c Department of Electrical Engineering-Physical Electronics, The Iby and Aladar Fleischman Faculty of Engineering, Tel Aviv University (Ramat Aviv 69978, Israel)
\end{abstract}

Received November 14, 2006 ; Accepted February 6, 2007

\begin{abstract}
The filling of trenches in ULSI interconnect structure by electroless copper deposition was investigated for the effect of bath additives. The additive effect was found to depend strongly on the reducing agent used in the bath. Void-free trench-filling was achieved by using polyethylene glycol (PEG) as an inhibiting additive in the bath containing glyoxylic acid as the reducing agent, while the combined addition of 8-hydroxy-7-iodo-5-quinoline sulfonic acid (HIQSA) and PEG was necessary for achieving void-free filling in the bath containing formaldehyde as the reducing agent. The effect of PEG on trench filling in the former bath was studied in detail based on electrochemical measurements. It is suggested that the rinse water remaining in trenches before electroless deposition causes a decrease in PEG concentration at the trench bottom during copper filling. The addition of PEG was found to shift the deposition potential in the negative direction. A new potential measuring apparatus was devised and used in model experiments, which revealed that the deposition potential depends on the local concentration of PEG at the trench bottom, where it is expected to be low. The observed preferential growth of copper deposit at the trench bottom is thus attributed to the effects of the variation of PEG concentration within the trenches on the deposition rate and potential.
\end{abstract}

Key Words : Electroless Deposition, Copper, Additive, ULSI Interconnects

\section{Introduction}

Electrodeposition of copper is used in the damascene process, ${ }^{1)}$ which is a process for fabricating interconnects in ultra large-scale integration of electronic circuits and components. In this process, submicrometer trenches and via-holes are filled with copper by electrodeposition, followed by chemical mechanical polishing. The electrodeposition process achieves the so-called "superfilling", which signifies filling of trenches and viaholes with copper without producing voids and/or seams. Several bath additives such as a deposition accelerating additive, a deposition inhibiting additive, and a leveling additive are used for successful achievement of this process. ${ }^{2-5)}$ Among these additives, accelerating and inhibiting additives are particularly important. These additives allow the so-called "bottom-up" growth to take place, which is the growth of copper deposit in the upward direction from the bottom of trenches, resulting in "superfilling". The bottom-up growth has been explained by a model ${ }^{6-9)}$ which is based on the assumption that the coverage with the accelerating additive at the trench bottom is greater than that at the trench opening during the deposition. In this model, the inhibiting additive decreases the deposition rate at the trench opening as a result of competitive adsorption with the accelerating additive. Although the electrodeposition process achieves superfilling, some critical issues, such as the need for the deposition of a uniform seed layer of sputtered copper and the problem of uneven current distribution on the wafer, still remain to be addressed.

The filling of trenches and via-holes by electroless copper deposition is expected to be an effective alternative for dealing with these problems, because electroless deposition is, in principle, capable of forming a uniform thin film on large substrates with superior step coverage. Superfilling has already been demonstrated to be achievable in an electroless copper plating bath containing small amounts of those additives which are used in the electrodeposition bath. Shingubara et al. ${ }^{10-12)}$ reported that superfilling was achieved by using bis(3-sulfopropyl)disulfide (SPS) as an inhibiting additive. Other researchers ${ }^{13,14)}$ have also achieved the superfilling by using SPS-containing baths, although they reported that SPS accelerated copper deposition at very low concentrations. Recently, we investigated superfilling ${ }^{15)}$ with a conventional electroless plating bath containing formaldehyde as the reducing agent, and demonstrated that superfilling was achievable by using the combination of 8-hydroxy-7-iodo-5-quinoline sulfonic acid $(\text { HIQSA })^{16)}$ as an accelerating additive and polyethylene glycol (PEG) as an inhibiting additive. Although superfilling was achieved in this bath, some modification of the 
bath composition was required for practical reasons. Namely, this bath contained formaldehyde as the reducing agent, which is undesirable for environmental reasons, and it also contained a large amount of sodium ions from the $\mathrm{pH}$ adjusting agent added as sodium hydroxide $(\mathrm{NaOH})$, which is known to adversely affect properties of transistors. Therefore, the present investigation was performed with a bath containing glyoxylic acid ${ }^{17,18}$ as an alternative to formaldehyde and tetramethyl ammonium hydroxide (TMAH) ${ }^{19)}$ as the $\mathrm{pH}$ adjusting agent instead of $\mathrm{NaOH}$. It was expected that the use of the different reducing agent might modify the additive effects, because both anodic and cathodic partial reactions comprising a full electroless deposition reaction are generally known to be affected by bath additives..$^{20,21)}$ Furthermore, other bath constituents might also affect partial as well as overall electroless deposition reactions. For example, Nakano et al. reported that the ease of electroless nickel deposition was strongly affected by replacing the alkaline salts in the bath with ammonium salts. ${ }^{19)}$ Thus, the use of an alternative $\mathrm{pH}$ adjusting agent was also thought to change the effect of additives on trench-filling in the present study. To clarify effects of these bath constituents on the bath performance, we examined effects of PEG and HIQSA on the trench-filling of patterned substrates, and carried out deposition rate measurements on unpatterned substrates. The results were compared with those obtained in the bath containing formaldehyde and sodium ions. Electrochemical polarization measurements and deposition potential measurements were also carried out to gain mechanistic understanding of the additive effect.

\section{Experimental}

The effect of additives on trench filling with electrolessly deposited copper was investigated by cross-sectional microscopic observation of copper-filled trenches combined with electrochemical measurements and deposition rate measurements.

Table 1 lists the compositions of three different baths, Baths A, B and C, used in this study. All baths were operated at $70{ }^{\circ} \mathrm{C}$ and a pH of 12.5 .

For trench-filling studies, $\mathrm{SiO}_{2} / \mathrm{Si}$ substrates with trench arrays were employed (Fig. 1). The trenches were $130 \mathrm{~nm}$ wide and $350 \mathrm{~nm}$ deep. A layer of tantalum metal and a copper catalytic layer were sputtered onto the substrates. The thickness of this $\mathrm{Cu} / \mathrm{Ti}$ layer on the side walls of trenches was approximately $35 \mathrm{~nm}$. The patterned substrates were pretreated with 5 vol\% $\mathrm{H}_{2} \mathrm{SO}_{4}$, rinsed in ultra pure water, and immersed into the plating bath. After copper deposition, the specimens were cut across the thickness using focused ion beam (FIB, FB-2100, Hitachi). The filling feature in cross-sectioned trenches was examined by a field emission scanning electron microscope (FE-SEM, S-4800, Hitachi).

The deposition rate of copper from these baths was measured gravimetrically on an unpatterned substrate with a $\mathrm{Cu}$ catalytic layer on a $\mathrm{Ti}$ adhesion layer, both of which were formed by physical vapor deposition. This substrate was activated by immersing into 5 vol\% of $\mathrm{H}_{2} \mathrm{SO}_{4}$ and rinsing with ultra pure water before the electroless copper deposition was carried out.

Electrochemical polarization measurements were performed with the complete electroless plating baths containing various concentrations of PEG. Polarization curves were recorded with a copper rotating disk electrode system (RRDE-1, Nikko Keisoku) equipped with a computer-controlled measuring system (HZ-3000, Hokuto Denko). A Cu disk working electrode measuring $0.6 \mathrm{~cm}$

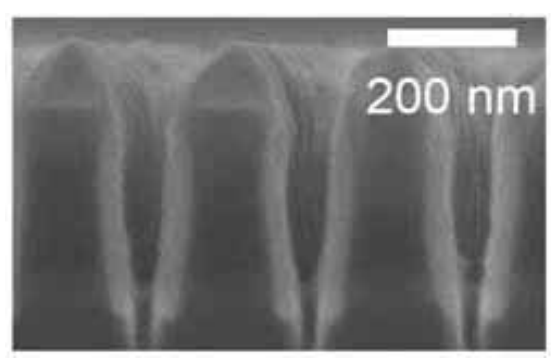

Fig. 1 A cross-sectional SEM image of trenches before electroless deposition.

Table 1 Bath compositions.

\begin{tabular}{lccc}
\hline & Bath A & Bath B & Bath C \\
\hline $\mathrm{CuSO}_{4} \cdot 5 \mathrm{H}_{2} \mathrm{O}$ & $0.04 \mathrm{~mol} \mathrm{~L}^{-1}$ & $0.04 \mathrm{~mol} \mathrm{~L}^{-1}$ & $0.04 \mathrm{~mol} \mathrm{~L}^{-1}$ \\
formaldehyde $(\mathrm{HCHO})$ & $0.04 \mathrm{~mol} \mathrm{~L}^{-1}$ & $0.04 \mathrm{~mol} \mathrm{~L}^{-1}$ & - \\
glyoxylic acid & - & - & $0.08 \mathrm{~mol} \mathrm{~L}^{-1}$ \\
ethylenediaminetetraacetic acid (EDTA) & $0.08 \mathrm{~mol} \mathrm{~L}^{-1}$ & $0.08 \mathrm{~mol} \mathrm{~L}^{-1}$ & $0.08 \mathrm{~mol} \mathrm{~L}^{-1}$ \\
pH & 12.5 & 12.5 & 12.5 \\
pH adjusting agent & $\mathrm{NaOH}$ & $\mathrm{TMAH}^{*}$ & $\mathrm{TMAH}^{*}$ \\
$\mathrm{PEG}^{*}(\mathrm{Mw} \mathrm{4000)}$ & $0,1 \mathrm{ppm}$ & $0,1 \mathrm{ppm}$ & $0,1 \mathrm{ppm}$ \\
$\mathrm{HIQSA}^{* * *}$ & $0,3 \mathrm{ppm}$ & $0,3 \mathrm{ppm}$ & $0,3 \mathrm{ppm}$ \\
\hline
\end{tabular}

Bath temperature: $70{ }^{\circ} \mathrm{C}$

* tetramethyl ammonium hydroxide

** polyethylene glycol

*** 8-hydroxy-7-iodo-5-quinoline sulfonic acid 
in diameter was polished first with \#2000 emery paper and subsequently with $0.06 \mu \mathrm{m}$ alumina paste on a sheet of polishing cloth. A Pt counter electrode and an $\mathrm{Ag} / \mathrm{AgCl}$ reference electrode were placed with the working electrode in a three-electrode cell. The RDE was rotated at a speed of $100 \mathrm{rpm}$. The measurements were performed at the potential scan rate of $10 \mathrm{mV} \mathrm{s}^{-1}$. Before each experiment, the bath solution was aerated to maintain the bath stability.

The effect of PEG on the deposition potential was investigated on both patterned and unpatterned substrates. The potential during immersion of the substrate into the bath was measured with the same measuring system as described above. The results on patterned and unpatterned substrates were compared and discussed. To consider the effect of concentration distribution of PEG, which was expected to be produced during trench filling, we also conducted another set of experiments consisting of potential measurements. The apparatus constructed for this set of experiments is illustrated in Fig. 2. Two electroless copper plating baths (Baths 1 and 2) were prepared, placed in separate vessels, and connected to each other through a salt bridge, which was a glass capillary filled with the basic bath solution without additives. In this study, two kinds of setups, namely Setups A and B were employed to examine the effect of the location of the salt bridge. In Setup A (Fig. 2a), an $\mathrm{Ag} / \mathrm{AgCl}$ reference electrode was connected to Bath 2 through the salt bridge. On the other hand, in
Setup B (Fig. 2b), the reference electrode was connected to Bath 1. A strip of $\mathrm{Cu}$ foil was employed as the working electrode. The two ends of this $\mathrm{Cu}$ foil were immersed into Baths 1 and 2, respectively. Measured amounts of an aqueous solution of PEG were added to these baths, while the potential of the $\mathrm{Cu}$ strip electrode was monitored during the process.

\section{Results and Discussion}

3. 1 Effect of additives on the performance of Baths $\mathrm{A}, \mathrm{B}$, and $\mathrm{C}$

Trench filling studies were performed with Baths A, B and $\mathrm{C}$ without additives, with PEG, and with both PEG and HIQSA. The effects of PEG and HIQSA in these three baths were examined by deposition rate.

3. 1. 1 Observation of trench filling First, the effects of PEG and HIQSA on trench filling in Bath A were investigated. Figure 3 shows cross-sectional SEM images of copper deposits obtained from baths without additives (additive-free bath, Fig. 3a), and with PEG alone (PEG bath, Fig. $3 \mathrm{~b}$ ), and with both PEG and HIQSA (PEG-HIQSA bath, Fig. 3c). Void-free filling was achieved in the PEG-HIQSA bath (Fig. 3c), while voids were clearly present in the specimens prepared in the additive-free (Fig. 3a) and PEG (Fig. 3b) baths. As was reported previously, ${ }^{15)}$ the addition of neither PEG nor HIQSA alone achieved void-free filling of trenches. It is apparent that the combined effect of PEG and HIQSA was necessary for the void-free filling.
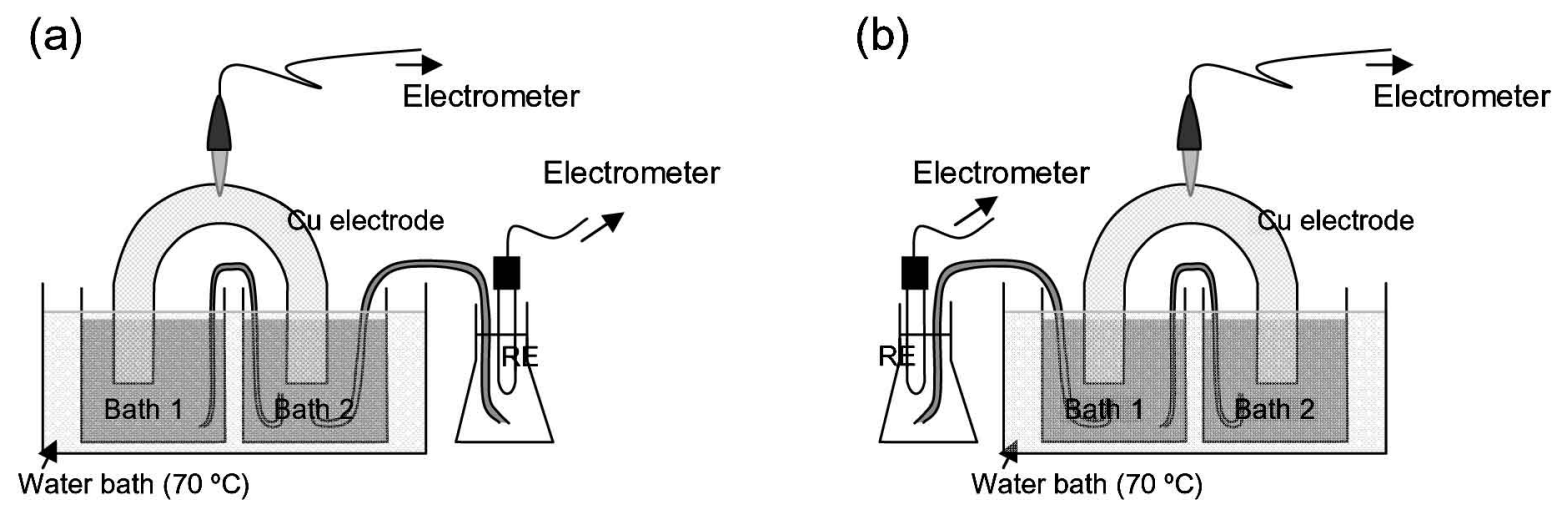

Fig. 2 Schematic illustration of the setups used for model experiments involving potential measurements in electroless copper baths with different compositions and activities: (a) Setup A and (b) Setup B.
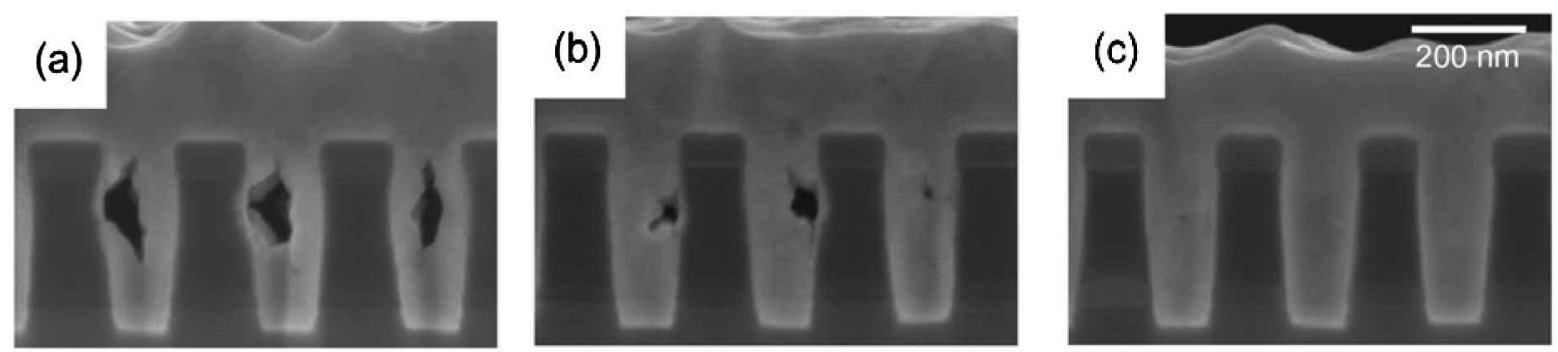

Fig. 3 Cross-sectional SEM images of copper deposited in trenches from (a) additive-free, (b) PEG, and (c) PEG-HIQSA baths. The basic bath composition was that of Bath A. Concentrations of PEG and HIQSA were 1 and 3 ppm, respectively. Deposition time, 3 min. 

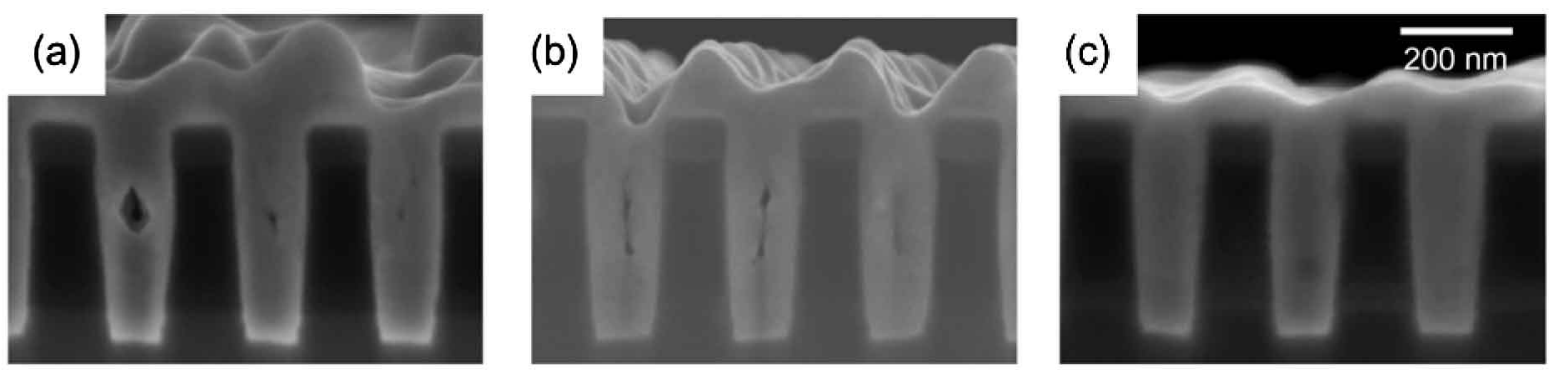

Fig. 4 Cross-sectional SEM images of copper deposited in trenches from (a) additive-free, (b) PEG, and (c) PEG-HIQSA baths. The basic bath composition was that of Bath B. Concentrations of PEG and HIQSA were 1 and 3 ppm, respectively. Deposition time, 3 min.
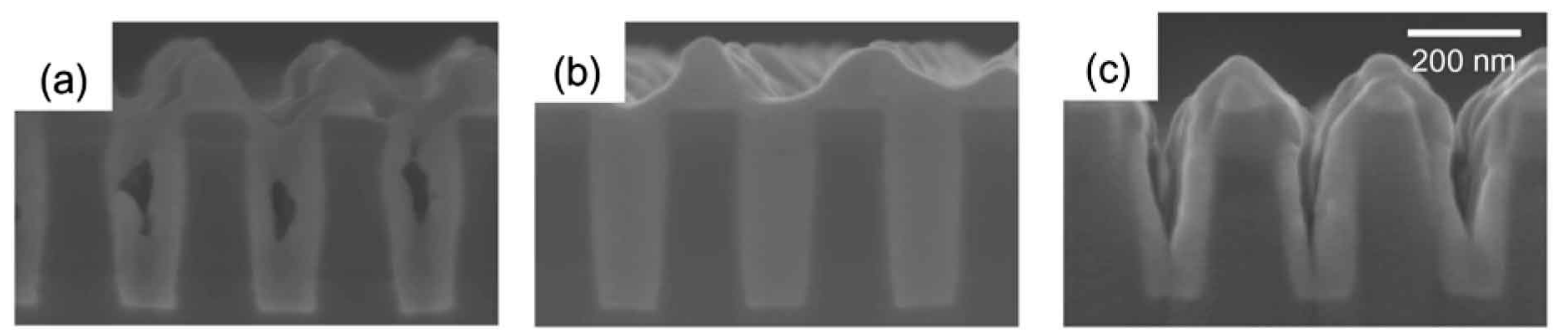

Fig. 5 Cross-sectional SEM images of copper deposited in trenches from (a) additive-free, (b) PEG, and (c) PEG-HIQSA baths. The basic bath composition was that of Bath C. Concentrations of PEG and HIQSA were 1 and 3 ppm, respectively. Deposition time, $3 \mathrm{~min}$.

To examine the effect of $\mathrm{pH}$ adjusting agent, a trenchfilling study was performed with Bath $\mathrm{B}$, which contained HCHO as the reducing agent and TMAH as the $\mathrm{pH}$ adjusting agent. The effects of PEG and HIQSA in this bath (Fig. 4) were similar to those in Bath A (Fig. 3). As can be seen in the images, voids were present in the specimens from the additive-free (Fig. 4a) and PEG (Fig. 4 b) baths. On the other hand, voids were not observed in the specimen prepared in the PEG-HIQSA bath (Fig. 4c). These results show that the combined effect of PEG and HIQSA appears irrespective of which $\mathrm{pH}$ adjusting agent is used.

In contrast, the effect of the additives was found to depend strongly on the reducing agent used in the bath. The cross sectional images of copper-filled trenches formed in Bath $\mathrm{C}$ are shown in Fig. 5. This bath contained glyoxylic acid as the reducing agent and TMAH as the $\mathrm{pH}$ adjusting agent. With this bath, superfilling was achieved when PEG was added alone (Fig. 5b). The effect of HIQSA was also found to depend on the reducing agent. For the PEG-HIQSA bath, trench filling was incomplete after the deposition time of $3 \mathrm{~min}$ (Fig. $5 \mathrm{c}$ ), whereas the trenches were completely filled with copper after the same deposition time in the PEG bath (Fig. 5b).

Copper was deposited in trenches with Bath $\mathrm{C}$ containing various concentrations of PEG (Fig. 6). It is seen that the effectiveness of trench-filling strongly depends on the concentration of PEG. It deteriorated at the higher concentrations of PEG. As shown in Figs. 6c and 6d, voids were present in all trenches filled with copper deposits from the bath containing more than $5 \mathrm{ppm}$ of PEG.
Figure $7 \mathrm{a}$ shows the evolution with time of the filling feature in trenches obtained in the bath containing 1 ppm of PEG. The result clearly shows that the deposition at trench openings was very slow, while that at the bottom was much faster, as discussed elsewhere. ${ }^{22)}$ To estimate the difference in deposition rate at the trench opening and at the bottom, the thickness of copper deposit at the trench opening $\left(\mathrm{T}_{\text {opening }}\right)$ and that at the bottom $\left(\mathrm{T}_{\text {bottom }}\right)$ were measured from the cross sections
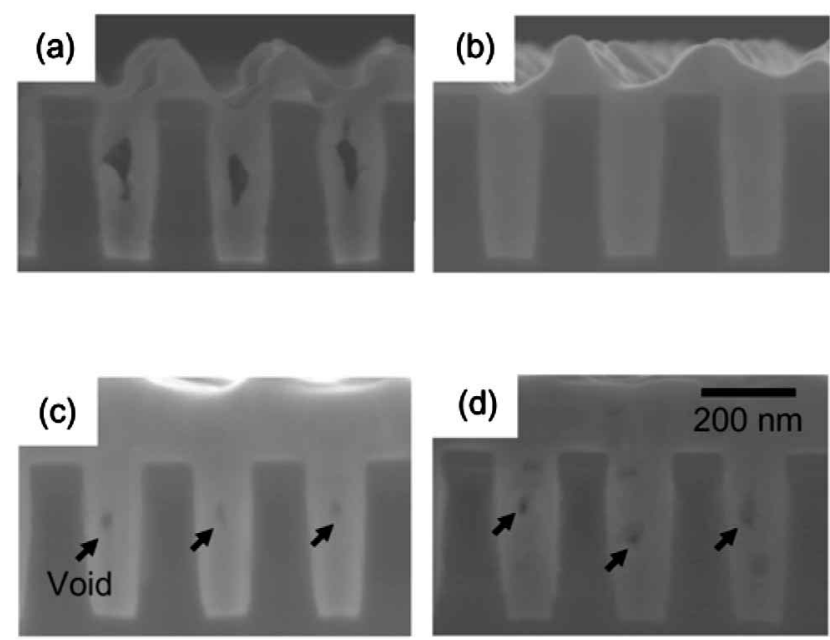

Fig. 6 Cross-sectional SEM images of copper deposited in trenches from (a) additive-free and (b-d) PEG baths. The basic bath composition was that of Bath C. PEG concentrations were (a) 0 , (b) 1 , (c) 5 , and (d) $10 \mathrm{ppm}$. Deposition time, (a and b) $3 \mathrm{~min}$, and (c and d) $15 \mathrm{~min}$. 
(a)
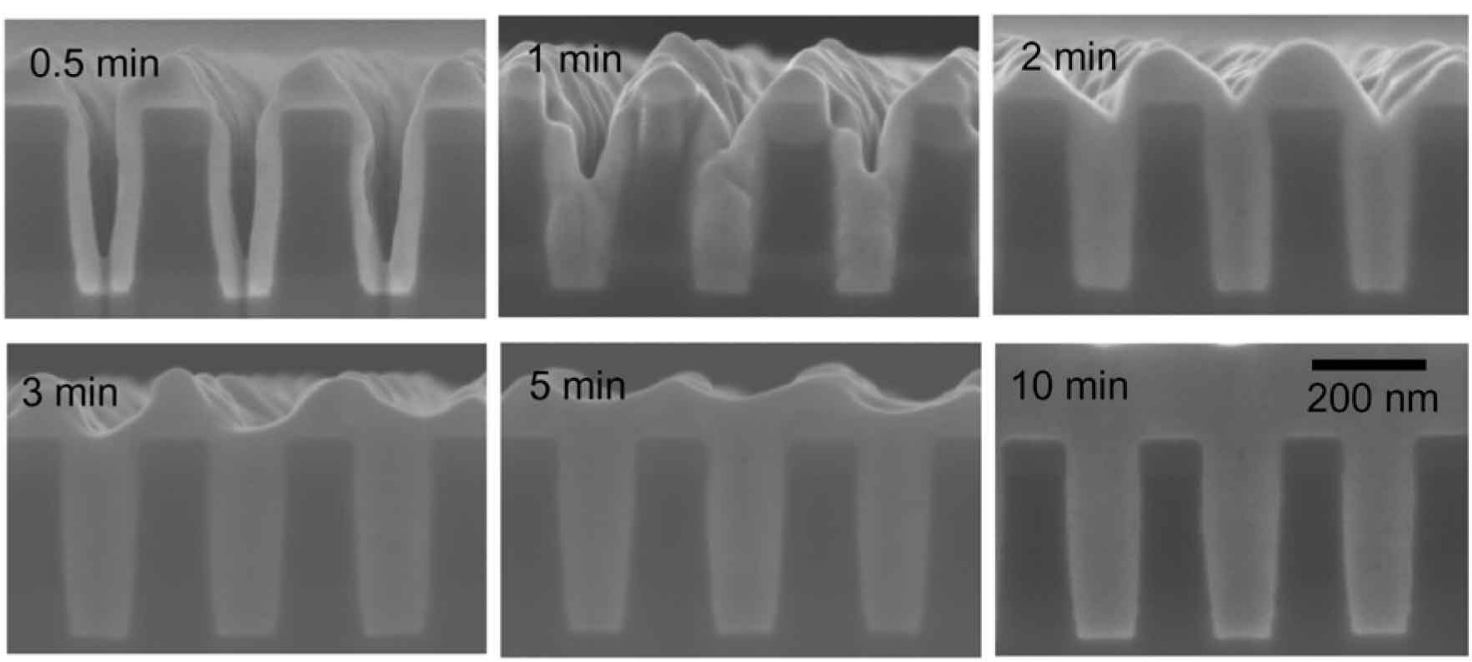

(b)

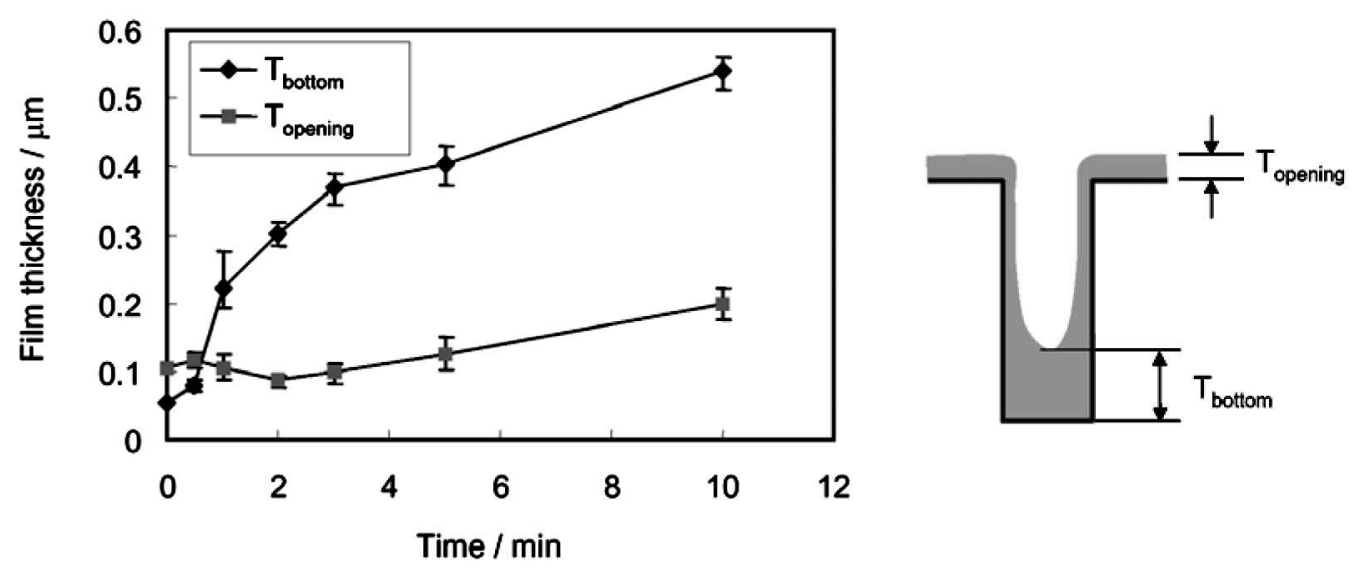

Fig. 7 (a) SEM images showing the change with time of cross-sectional characteristics of copper deposited in trenches from PEG bath. The basic bath composition was that of Bath C. Concentration of PEG was 1 ppm. Deposition time was varied from 0.5 to $1,2,3,5$, and $10 \mathrm{~min}$. (b) Change with time of the thickness of copper deposits at bottom (T $\mathrm{T}_{\text {botom }}$ ) and opening $\left(\mathrm{T}_{\text {opening }}\right.$ ) of trenches. The data were obtained from the SEM images.

shown in Fig. 7a, and the results were plotted against deposition time (Fig. $7 \mathrm{~b}$ ). It is interesting to note that the thickness of copper in the area of trench openings $\left(\mathrm{T}_{\text {opening }}\right)$ remained almost constant during the period of trench filling (0-5 min), while that at the bottom ( $\left.\mathrm{T}_{\text {bottom }}\right)$ increased significantly with time. The deposition rates at the bottom, estimated from Fig. 7b, were more than $10 \mu \mathrm{m} / \mathrm{hr}$ during filling process $(0.5-1 \mathrm{~min})$. After the trenches were filled with copper (5-10 min), the thickness at the opening began to increase. In this period, the rate of the increase in film thickness at the openings was essentially identical to that at the bottom, i.e., both rates were approximately equal to $1 \mu \mathrm{m} / \mathrm{hr}$. These results clearly show that a significant bottom-up growth occurred in this bath at the early stages of the deposition. The observed filling behavior is a demonstration of superfilling by electroless copper deposition. It is particularly interesting to note that the difference in the depo- sition behavior at the bottom and at the opening of trenches is assumed to be due to the difference in the concentration of PEG at these different portions of trenches. The mechanism of trench filling by PEG observed in Bath $\mathrm{C}$ is discussed further in the latter section.

3. 1. 2 Deposition rate measurements To gain insight into the effect of those additives on copper deposition, deposition rate measurements were carried out on the unpatterned substrate. The rate of copper deposition measured in Bath $\mathrm{A}$ without additives (additive-free bath) was $6.48 \mu \mathrm{m} / \mathrm{hr}$, and it decreased by $15 \%$ when PEG was added, while the addition of HIQSA increased the rate by more than $50 \%$. The deposition rate for the PEG-HIQSA bath was $5.75 \mu \mathrm{m} / \mathrm{hr}$, which was almost the same as that of the PEG bath, which was $5.65 \mu \mathrm{m} / \mathrm{hr}$. We reported previously that the acceleration effect of HIQSA in the PEG-HIQSA bath was significant only at 
very low concentrations, and that this concentration dependence of deposition rate is considered responsible for achieving the void-free filling. ${ }^{15)}$ In Bath $\mathrm{B}$, the effect of PEG and HIQSA is similar to that in Bath A. This result agreed well with the result of trench filling studies.

For Bath $\mathrm{C}$, the effects of these additives on the rate of copper deposition were quite different from those in Baths A and B. The inhibition effect of PEG in Bath C was more significant than that in Baths A and B. The rate of copper deposition for Bath C containing PEG was $1.08 \mu \mathrm{m} / \mathrm{hr}$, which is as much as $85 \%$ lower than that of the additive-free bath $(7.45 \mu \mathrm{m} / \mathrm{hr})$. The decrease in the rate of copper deposition with an increase in PEG concentration was significant only at the concentrations lower than $1 \mathrm{ppm}$. On the other hand, the rate of copper deposition became constant at concentrations higher than $1 \mathrm{ppm}$. It should be noted that this deposition rate was nearly the same as that on the patterned substrate in the PEG $(1 \mathrm{ppm})$ bath, which was estimated from Fig. $7 \mathrm{~b}$, during the period after the trench was filled with copper (5-10 min). The effect of HIQSA on the deposition rate of this bath was also different from that of Baths A and B. The rate of deposition decreased by 18 $\%$ with the addition of $3 \mathrm{ppm}$ of HIQSA, and it continued to decrease with an increase in HIQSA concentration. The results of deposition rate measurements described above revealed that the effects of both PEG and HIQSA depend strongly on the reducing agent, indicating that both additives affect the reaction of the reducing agent.

The dependency of the additive effect on the reducing agent described above explains the results of trench filling studies. Because the inhibition effect of PEG in Bath $\mathrm{C}$ is more significant than that in Baths $\mathrm{A}$ and $\mathrm{B}$, the difference in the rate of copper deposition between the trench opening and the trench bottom, which is believed to be caused by the decrease in PEG concentration at the bottom brought about by the slow diffusion of PEG (vide infra), should be more significant. As a result, the sufficient bottom-up growth was provided by adding PEG alone to Bath C. On the other hand, HIQSA did not accelerate but decelerate the deposition. Consequently, the filling property was not improved by the addition of HIQSA to the PEG bath in the case of Bath $\mathrm{C}$, and the rate of copper deposition within the trenches was decreased in this bath.

\section{2 Electrochemical investigation on the effect of PEG on trench filling in Bath C}

As described above, the addition of PEG to Bath C was found to be highly useful for achieving void-free filling. To investigate the effect of PEG in this bath, polarization measurements were carried out with a $\mathrm{Cu}$ rotating disk electrode. In addition, deposition potential measurements were performed on both patterned and unpatterned substrates. To obtain understanding of the mechanism of the effect of PEG on trench-filling, further investigation was carried out by using the potential measuring apparatus with a $\mathrm{Cu}$ strip electrode, which was explained in the preceding section (Fig. 2).
3. 2. 1 Electrochemical polarization measurements Electrochemical polarization measurements were carried out to investigate the effect of PEG on the kinetics of eletroless copper deposition in Bath C. Electroless deposition reactions in general have been investigated based on the mixed potential theory, ${ }^{23)}$ in which the oxidation of the reducing agent and the reduction of metal ions are assumed to occur simultaneously at the same rate on the substrate. According to this theory, superposition of polarization curves for the two partial reactions should yield the curve for the complete electroless bath. However, in the case of the $\mathrm{Cu}(\mathrm{II})$-EDTA electroless plating system with formaldehyde as the reducing agent, it has been reported that the polarization curve obtained with the complete bath did not correspond to the sum of the curves obtained separately for the anodic and cathodic partial reactions. ${ }^{24-26}$ ) One of the present author ${ }^{24)}$ compared the polarization curve for the complete bath with the curve for the solution in the absence of formaldehyde and with that for the solution in the absence of cupric ions. It was found that the reduction of $\mathrm{Cu}$ (II)-EDTA complex was greatly accelerated by the presence of formaldehyde, which suggested the existence of a strong interaction between formaldehyde and the copper complex. Because glyoxylic acid undergoes an oxidation reaction similar to that of formaldehyde, ${ }^{18)}$ the oxidation of the former reducing agent is also expected to interact with the reduction reaction of the $\mathrm{Cu}(\mathrm{II})$-EDTA complex. To investigate the effect of the interaction between the reducing agent and the copper complex on electroless copper deposition, electrochemical polarization measurements were performed with a complete bath containing both glyoxylic acid and $\mathrm{Cu}(\mathrm{II})$ EDTA complex (Fig. 8, curve 1), a bath containing no $\mathrm{Cu}(\mathrm{II})$ complex (Fig. 8, curve 2), and a bath containing no glyoxylic acid (Fig. 8, curve 3). These curves clearly showed that glyoxylic acid greatly accelerated the reduction of $\mathrm{Cu}(\mathrm{II})$-EDTA complex. Furthermore, the anodic current peak at $-0.5 \mathrm{~V}$ was also found to increase in the presence of $\mathrm{Cu}(\mathrm{II})$-EDTA complex. These curves show that the reduction of $\mathrm{Cu}(\mathrm{II})$-EDTA complex is accelerated by glyoxylic acid, and that the oxidation of glyoxylic acid is also accelerated in the presence of $\mathrm{Cu}(\mathrm{II})$-EDT A complex. In the next experiment, therefore, the effect of PEG on electroless deposition was investigated with the complete electoless bath in the presence of both the reducing agent and $\mathrm{Cu}(\mathrm{II})$-EDTA. Figure $9 \mathrm{a}$ shows the polarization curves recorded in the complete baths containing various concentrations of PEG. The anodic and cathodic current peaks at $-0.45 \mathrm{~V}$ and $-0.90 \mathrm{~V}$ vs. $\mathrm{Ag} / \mathrm{AgCl}$, are attributed to the oxidation of glyoxylic acid and the reduction of $\mathrm{Cu}(\mathrm{II})$-EDTA complex, respectively. The current densities at both peaks decreased with an increase in PEG concentration. This indicates that both the anodic and cathodic reactions were suppressed by PEG. The addition of PEG was also found to affect the deposition potential. Figure $9 \mathrm{~b}$ shows a plot of copper deposition potential, which is a zero current potential in the polarization curve, against PEG concentration. The deposition potential in the additive-free 


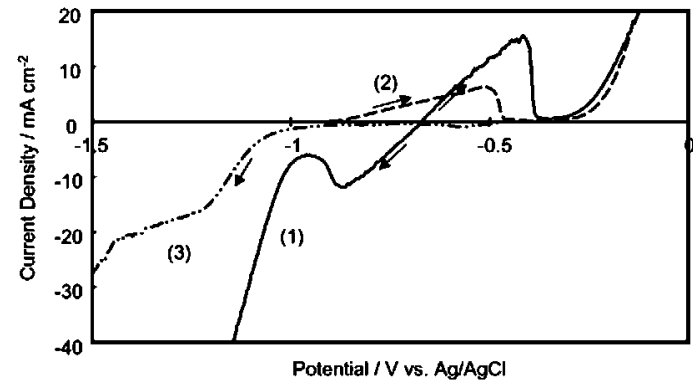

Fig. 8 Polarization curves for (1) the complete electroless copper bath containing both glyoxylic acid and cupric ions (Baths C), for (2) the bath containing no cupric ions, and for (3) the bath containing no glyoxylic acid.

(a)

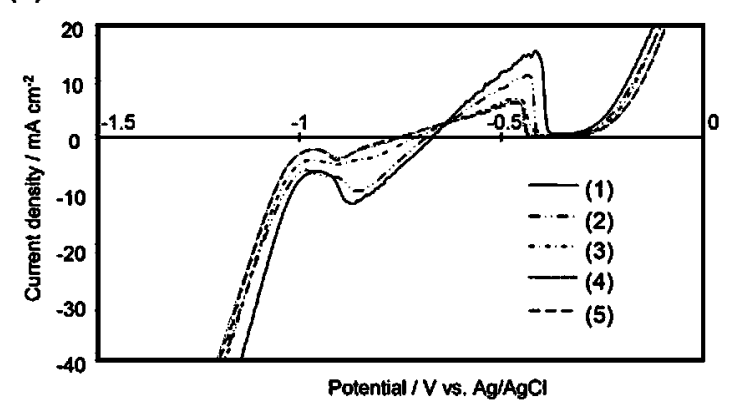

(b)

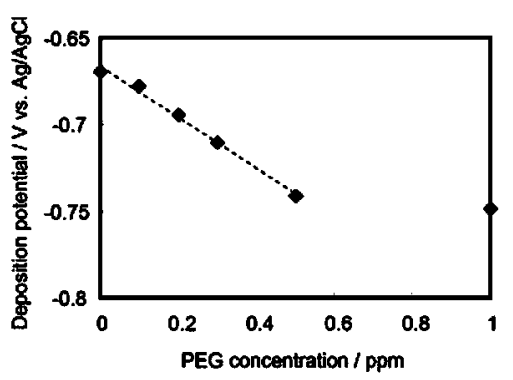

Fig. 9 (a) Polarization curves for Bath $\mathrm{C}$ with additions of (1) 0 , (2) 0.1, (3) 0.3, (4) 0.5, and (5) 1 ppm of PEG. (b) Effect of PEG on the deposition potential obtained from polarization curves.

(PEG-free) bath was $-0.67 \mathrm{~V}$ vs. $\mathrm{Ag} / \mathrm{AgCl}$. It shifted in the negative direction with an increase in PEG concentration in the range of 0 to $0.5 \mathrm{ppm}$. The deposition potential was almost constant in the presence of more than $0.5 \mathrm{ppm}$ of PEG, and the potential measured in the bath containing $1 \mathrm{ppm}$ of PEG was $-0.75 \mathrm{~V}$.

\section{2. 2 Deposition potential measurements The} polarization measurement showed that the addition of a greater amount of PEG shifted the potential for electroless copper deposition to a more negative value. For trench filling, the concentration of PEG at the bottom is expected to be lower than that at the opening. Thus, the effect of PEG on the deposition potential within the trenches is expected to be different from that observed on an unpatterned substrate. To verify this expectation, the potentials of trench-patterned substrates immersed into the additive-free and PEG baths were measured
(Fig. 10, curves a and b, respectively), and the results were compared with those measured on unpatterned substrates (Fig. 10, curves $c$ and d). Curves a and b in Fig. 10 were obtained with patterned substrates. For this measurement, the patterned substrate, which had just been activated with $\mathrm{H}_{2} \mathrm{SO}_{4}$ and rinsed with UPW, was immersed into the bath $(0 \mathrm{sec})$, and its potential was measured continuously during the period of copper deposition. It should be noted here that the substrate-pretreatment procedure immediately before the electroless deposition was the rinse in UPW. Because the trenches employed in this study were very small in diameter (130 $\mathrm{nm}$ ), the trenches were filled with UPW at the initial stages. The UPW in the trenches must have been replaced by the electroless plating solution before the electroless plating began to take place. For the first 12 sec, the potential measured in the additive-free bath (Fig. 10 , curve a) was less negative than $-0.5 \mathrm{~V}$, where copper deposition did not proceed, while it shifted to more negative values with time during this period. At $12 \mathrm{sec}$ the potential abruptly shifted from $-0.47 \mathrm{~V}$ to $-0.67 \mathrm{~V}$. This initial period of $12 \mathrm{sec}$ was regarded as the induction period involved in the overall electroless copper deposition. After the induction period was over, the deposition potential became almost constant at $-0.68 \mathrm{~V}$, for at least 2 min. Compared to this result obtained with the additivefree bath, the potential-time curve recorded with a patterned substrate in the bath containing $1 \mathrm{ppm}$ of PEG (Fig. 10, curve b) was quite different, although the results were similar to each other for the initial induction period of $12 \mathrm{sec}$. The potential of the PEG bath also shifted significantly at $12 \mathrm{sec}$ in the negative direction. After the induction period was over, the deposition potential for the PEG (1 ppm) bath shifted to $-0.7 \mathrm{~V}$ (Fig. 10 , curve b, $12 \mathrm{sec}$ ), which was similar to the deposition potential of the unpatterned substrate in the additivefree (PEG-free) bath (Fig. 10, curve c). Unlike for the additive-free bath, the potential for the PEG bath (Fig. 10 , curve b) continued to shift gradually from $-0.70 \mathrm{~V}$ to $-0.76 \mathrm{~V}$ during the period of 12 to $70 \mathrm{sec}$, at which time it became constant. The potential measured with the patterned substrate after $70 \mathrm{sec}$ was $-0.76 \mathrm{~V}$ (Fig. 10, curve b). It should be noted that this potential was essentially identical to that measured on the unpatterned substrate in the PEG (1 ppm) bath (Fig. 10, curve d). The timedependence of the potential of the unpatterned substrate (Fig. 10, curves $\mathrm{c}$ and d) in both additive-free and PEG baths was different from that of the patterned substrate (Fig. 10, curves a and b). The induction period of initial $12 \mathrm{sec}$ associated with the patterned substrate was not observed with the unpatterned substrate. The potential of the unpatterned substrate in the additive-free bath (Fig. 10, curve c) and that in the PEG bath (Fig. 10, curve d) shifted significantly in the negative direction within the initial period of only $1.5 \mathrm{sec}$. After this period of time, the potential values in the two baths reached -0.7 $\mathrm{V}$ and $-0.76 \mathrm{~V}$, respectively, after which these values remained steady and independent of time. It is important to note that the potential shift after the induction period observed in the PEG $(1 \mathrm{ppm})$ bath with the pat- 

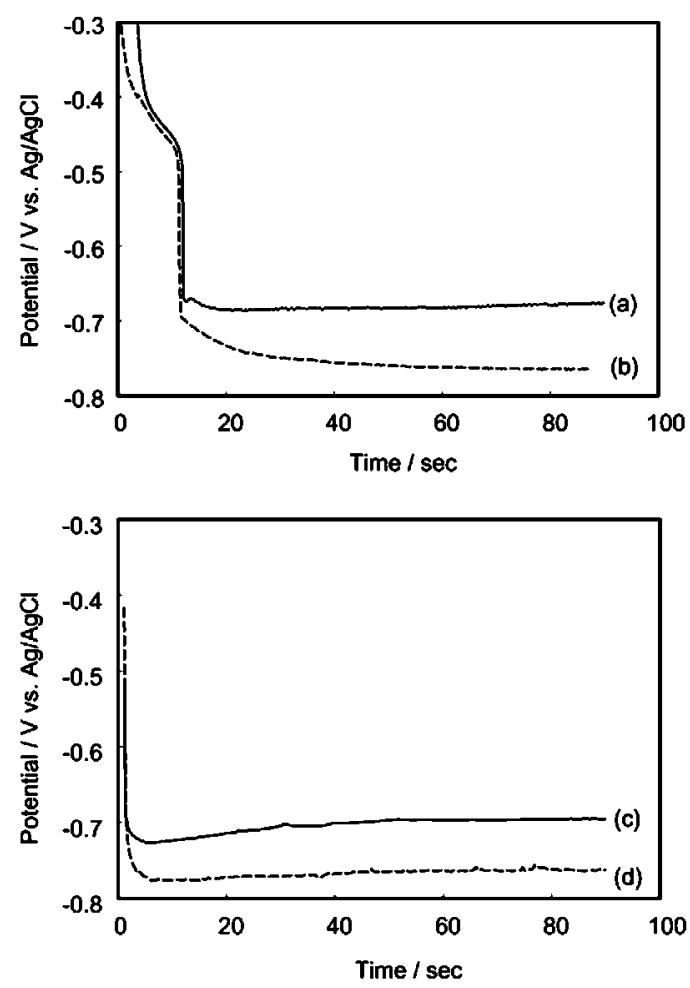

Fig. 10 Change with time of the potential of (a and b) a patterned substrate and (c and d) an unpatterned substrate during electroless copper deposition. The basic bath composition was that of Bath C. Measurements were carried out in (a and c) additive-free bath, and ( $b$ and d) PEG bath. Concentration of PEG, 1 ppm.

terned substrate (Fig. 10, curve b, 12-70 sec), was not found with the unpatterned substrate (Fig. 10d). The negative shift of the potential for the PEG bath observed after the induction period as well as the presence of induction period was characteristic of the patterned substrate. The observed change in the potential in the PEG (1 ppm) bath measured with the patterned substrate is attributed to the change in PEG concentration in trenches during the initial diffusion process of PEG. The increase in the concentration of PEG, which is a large polymer molecule, is assumed to be slow inside the trenches, where the mass transfer of bath constituent species is greatly restricted. Thus, the concentration of PEG at the trench bottom must have been lower than that at the opening during trench filling. We believe that the observed difference in the deposition behavior at the bottom and at the opening (Fig. 7b) is due to the difference in PEG concentration at the two different locations.

To understand the effect of concentration distribution of PEG on a substrate on the copper deposition potential in greater detail, we carried out a series of simple model experiments using the apparatus illustrated in Fig. 2a (Setup A), where the reference electrode was connected to Bath 2 through a salt bridge. Two PEG-free baths (Baths 1 and 2) were prepared for the experiment. Each end of a $\mathrm{Cu}$ foil electrode was immersed into either of the two baths $(0 \mathrm{sec})$, and the potential of each end of the foil was measured against the reference electrode (The duration in which these potentials were measured is designated as the "first period"). For the second period, an aqueous solution of PEG was added to Bath 1 (45 sec) to make the PEG concentration in the bath equal to $1 \mathrm{ppm}$. For the third period, the same amount of the PEG solution was added to Bath $2(70 \mathrm{sec})$. The variations of electrode potential during these three distinct periods of time are shown in Fig. 11. When neither of the two baths contained PEG (first period), the deposition potential was approximately equal to $-0.70 \mathrm{~V}$, which was identical to the deposition potential for the additivefree bath (Fig. 10, curve c). The PEG solution was then added only to Bath 1 at 45 sec. In view of the observed effect of PEG on the deposition potential shown in Fig. $9 \mathrm{~b}$, the potential of the $\mathrm{Cu}$ foil electrode was expected to change upon the addition of PEG to Bath 1 . On the contrary, however, the potential remained unchanged when PEG was added only to Bath 1 (the second period). On the other hand, when PEG was added also to Bath 2 (70 sec), the deposition potential gradually shifted in the negative direction to $-0.76 \mathrm{~V}$, which was almost identical to the deposition potential found with the bath containing 1 ppm of PEG. In the experiment performed with Setup A described above, the potential of the $\mathrm{Cu}$ strip electrode was measured against the reference electrode, which was connected to Bath 2 through the salt bridge. To examine the effect of the location of the salt bridge, the same measurement was performed with Setup B, where the reference electrode was connected to Bath 1 (Fig. $2 b)$. The procedure of the potential measurement was almost the same as that performed with Setup A (Fig. 11a). For this measurement with Setup B, two PEG-free baths (Baths 1 and 2) were prepared, and each end of a $\mathrm{Cu}$ foil electrode was immersed into either of the two baths (0-85 sec, the "first period" in Fig. 11b). At $85 \mathrm{sec}$, the PEG solution was added to Bath 1 (85-130 sec, the "second period" in Fig. 11b). The result of this measurement (Fig. 11b) clearly shows that the potential was unchanged with the addition of PEG to Bath 1 , and that the potential was kept at $-0.7 \mathrm{~V}$, which was identical to the deposition potential in the additive-free bath measured with an unpatterned substrate. These results show that the potential of the $\mathrm{Cu}$ strip electrode was independent of the location of the salt bridge. It is thus concluded that the copper deposition at an electrode with two distinctly separated areas, i.e., with one area contacting the PEG-free bath and the other area contacting the PEG-containing bath, was controlled by the reaction occurring at the interface between the copper surface and the PEG-free bath. This result also suggests that the deposition occurs at the potential corresponding to the deposition potential assumed by that portion of the substrate where the deposition potential was less negative.

The shift of potential measured with the patterned substrate during the progress of the electroless deposition reaction in the PEG (1 ppm) bath (Fig. 10, curve b) is considered to have resulted from the decrease in PEG concentration within the trenches, which, in turn, was 
(a)

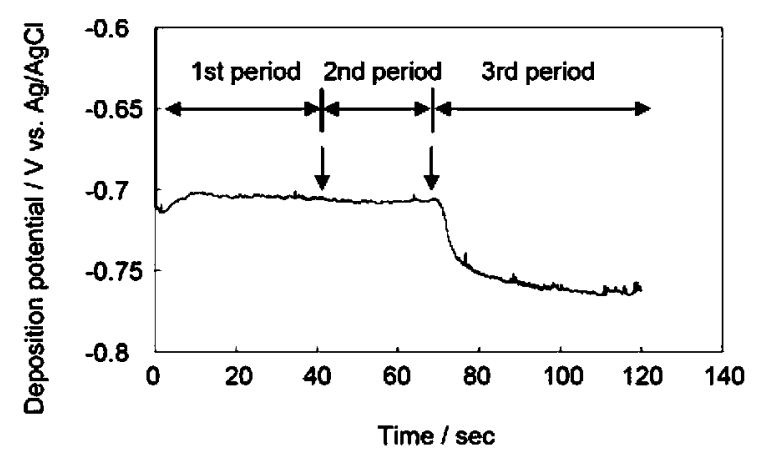

(b)

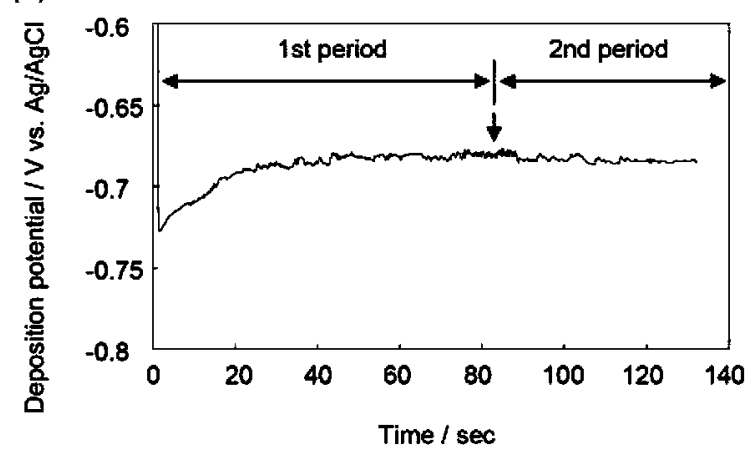

Fig. 11 Change with time of the potential of a copper strip electrode with two ends immersed into Baths 1 and 2, respectively. The basic bath composition was that of Bath C. Measurements were performed with (a) Setup A and (b) Setup B (see Figs. 2a and 2b).

caused by the slow diffusion of these species from the bulk of the solution. The concentration of PEG, which is a large polymer molecule, is expected to decrease toward the trench bottom, where the diffusion of PEG from the bulk of the solution is restricted for geometrical reasons. As suggested by the result shown in Fig. 11, the deposition potential is controlled by the concentration of PEG at that portion of the electrode which is in contact with the solution where PEG concentration is the lowest. If the concentration gradient of PEG thus produced in trenches is taken into consideration, the deposition potential during trench filling is expected to be determined by the PEG concentration at the bottom of the trench. The diffusion of PEG from the bulk of the solution into trenches and the change in the feature of trench filling during the copper deposition appear to lead to an increase in the concentration of PEG at the bottom. As a result, the difference in deposition rate at the bottom and at the opening should become less significant with time.

We will now discuss the effect of PEG on trench filling based on the results of potential measurements presented above. If the concentration of PEG at the bottom is nil, while that at the opening is $1 \mathrm{ppm}$, the deposition potential during trench filling should correspond to that of the additive-free (PEG-free) bath, which is approximately $-0.7 \mathrm{~V}$. Because this potential is less negative than that measured on an unpatterned substrate in the bath containing $1 \mathrm{ppm}$ of PEG $(-0.75 \mathrm{~V})$, the deposition rate at the trench openings in the PEG (1 ppm) bath should be lower than that on an unpatterned substrate in the same bath. Indeed, the deposition rate at this potential measured in the PEG bath was $0.72 \mu \mathrm{m} / \mathrm{hr}$, which was lower than that at the deposition potential of the PEG $(1 \mathrm{ppm})$ bath, which was $1.08 \mu \mathrm{m} / \mathrm{hr}$. This assumption was supported by the fact that the effect of PEG on the filling properties strongly depends on its concentration in the bath, which is shown by the SEM images in Fig. 6. The images show that void-free filling was achieved by the addition of $1 \mathrm{ppm}$ of PEG, while voids were formed in copper-filled trenches, at higher concentrations of PEG. The optimum concentration of PEG $(1 \mathrm{ppm})$ was within the range where the effects of PEG concentration on the inhibition of deposition and on the effect on the deposition potential were significant. On the other hand, at the concentrations higher than 1 $\mathrm{ppm}$, the effect of PEG was nearly independent of its concentration. Consequently, the difference in the deposition rate at the bottom and at the opening of trenches, which is caused by the PEG concentration gradient in trenches, is considered to be much less significant in this range of PEG concentreation, leading to the formation of voids.

\section{Conclusion}

The effect of PEG and HIQSA on the electroless copper deposition in trenches was investigated. Effects of reducing agent and $\mathrm{pH}$ adjusting agent on the behavior of the baths were also examined. The effect of the above additives strongly depended on the reducing agent. Void-free filling was achieved by adding PEG alone to the bath containing glyoxylic acid as the reducing agent, while the addition of both PEG and HIQSA was necessary in the bath containing formaldehyde as the reducing agent.

Electrochemical measurements were performed with the bath containing glyoxylic acid to investigate the effect of PEG on trench filling. The addition of PEG shifted the deposition potential in the negative direction, and it decreased the deposition rate significantly. The higher deposition rate at the bottom of trenches than that at the opening, observed during copper filling in the bath containing PEG, was attributed to the decrease in PEG concentration at the bottom, which results from the rinse water remaining in the trenches before the substrate was immersed in the bath. The effect of PEG on the deposition potential during trench filling was discussed by taking into account the local concentration distribution inside the trenches. The potential measurement carried out with a $\mathrm{Cu}$ strip electrode, each end of which was immersed in either of the two different baths with and without PEG connected through a salt bridge, revealed that the measured potential was identical to that assumed by a piece of copper immersed in a separate, additive-free bath. These results suggest that the deposition potential measured with a copper electrode consisting of portions immersed in a solution with locally different activities, assumes the value corresponding to 
the deposition potential of a copper electrode immersed in the solution with the highest activity. Under the conditions of trench filling, the potential of copper deposition is controlled by the local PEG concentration at the trench bottom, where the concentration of PEG is expected to be the lowest. It is concluded that the superfilling achieved by the addition of PEG is brought about by the shift of deposition potential resulting from the low PEG concentration at the trench bottom, combined with the inhibition effect of PEG at the trench opening.

\section{Acknowledgements}

This work was supported by the 21st Century COE Program of the Center for Practical Nano-Chemistry and by the Encouraging Development of Strategic Research Centers Program of the Consolidated Research Institute for Advanced Science and Medical Care, both granted by the MEXT, Japan.

\section{References}

1) P. C. Andricacos, C. Uzoh, J. O. Dukovic, J. Horkans, and H. Deligianni, IBM J. Res. Dev., 42, 567 (1998).

2) J. J. Kelly and A. C. West, Electrochem. Solid-State Lett., 2, 561 (1999).

3) P. Taephaisitphongse, Y. Cao, and A. C. West, J. Electrochem. Soc., 148, C492 (2001).

4) S. Miura, K. Oyamada, Y. Takada, and H. Honma, Electrochemistry, 69, 773 (2001).

5) M. Hasegawa, Y. Negishi, T. Nakanishi, and T. Osaka, J. Electrochem. Soc., 152, C221 (2005).

6) T. P. Moffat, J. E. Bonevich, W. H. Huber, A. Stanishevsky, D. R. Kelly, G. R. Stafford, and D. Josell, J. Electrochem. Soc., 147, 4524 (2000).

7) T. P. Moffat, D. Wheeler, C. Witt, and D. Josell, Electrochem. Solid-State Lett., 5, C110 (2002).

8) T. P. Moffat, D. Wheeler, M. D. Edelstein, and D. Josell,
IBM J. Res. Dev., 49, 19 (2005).

9) A. C. West, S. Mayer, and J. Reid, Electrochem. SolidState Lett., 4, C50 (2001).

10) S. Shingubara, Z. Wang, O. Yaegashi, R. Obata, H. Sakaue, and T. Takahagi, Electrochem. Solid-State Lett., 7, C78 (2004).

11) Z. Wang, O. Yaegashi, H. Sakaue, T. Takahagi, and S. Shingubara, J. Electrochem. Soc., 151, C781 (2004).

12) Z. Wang, O. Yaegashi, H. Sakaue, T. Takahagi, and S. Shingubara, Jpn. J. Appl. Phys., 43, 7000 (2004).

13) C. H. Lee, S. C. Lee, and J. J. Kim, Electrochim. Acta, 50, 3563 (2005).

14) C. H. Lee, S. C. Lee, and J. J. Kim, Electrochem. SolidState Lett., 8, C110 (2005).

15) M. Hasegawa, Y. Okinaka, Y. Shacham-Diamand, and T. Osaka, Electrochem. Solid-State Lett., 9, C138 (2006).

16) L. N. Schoenberg, J. Electrochem. Soc., 119, 1491 (1972).

17) J. Darken, Trans. Inst. Metal Finish., 69, 66 (1991).

18) H. Honma and T. Kobayashi, J. Electrochem. Soc., 141, 730 (1994).

19) H. Nakano, T. Itabashi, and H. Akahoshi, J. Electrochem. Soc., 152, C163 (2005).

20) M. Paunovic and R. Arndt, J. Electrochem. Soc., 130, 794 (1983).

21） A. Hung, J. Electrochem. Soc., 132, 1047 (1985).

22) M. Hasegawa, N. Yamachika, Y. Okinaka, Y. ShachamDiamand, and T. Osaka, Appl. Phys. Lett., submitted.

23) C. Wagner and W. Traud, Z. Elektrochem., 44, 391 (1938).

24) Y. Okinaka and T. Osaka, Advances in Electrochemical Science and Engineering, Vol. 3 (Eds. H. Gerischer and C. W. Tobias), VCH Publishers Inc., New York, NY, p.55 (1994); See also references cited therein.

25) B. J. Feldmann and O. R. Melroy, J. Electrochem. Soc., 136, 640 (1989).

26) H. Wiese and K. G. Weil, Ber. Bunsenges. Phys. Chem., 91, 619 (1987). 